\title{
A NON-ADDITIVE THERMODYNAMIC FORMALISM AND DIMENSION THEORY OF HYPERBOLIC DYNAMICAL SYSTEMS
}

\author{
LUIS M. BARREIRA
}

\begin{abstract}
A в STRACт. We develop a non-additive version of the thermodynamic formalism. This allows us to unify and extend many results of dimension theory of hyperbolic dynamical systems. We obtain lower and upper bounds for the dimension of a broad class of Cantor-like sets and hyperbolic sets.
\end{abstract}

\section{Introduction}

We begin with the description of a geometric construction in the real line. We consider $p$ positive numbers $\lambda_{1}, \ldots, \lambda_{p}<1$, and choose $p$ disjoint closed intervals $\Delta_{1}, \ldots, \Delta_{p}$ with length $\lambda_{1}, \ldots, \lambda_{p}$. For each $k=1, \ldots, p$, we choose again $p$ disjoint closed intervals $\Delta_{k 1}, \ldots, \Delta_{k p} \subset \Delta_{k}$ with length $\lambda_{k} \lambda_{1}, \ldots, \lambda_{k} \lambda_{p}$. Iterating this procedure, for each integer $n$ we obtain $p^{n}$ disjoint closed intervals $\Delta_{i_{1} \cdots i_{n}}$ with length $\prod_{k=1}^{n} \lambda_{i_{k}}$. We define the set

$$
F=\bigcap_{n=1}^{\infty} \bigcup_{\left(i_{1} \cdots i_{n}\right)} \Delta_{i_{1} \cdots i_{n}} .
$$

In $[\mathrm{M}]$, Moran showed that the Hausdorff dimension of $F$ is the unique root $s$ of the equation

$$
\sum_{k=1}^{p} \lambda_{k}^{s}=1 .
$$

This result does not depend on the location of the intervals $\Delta_{i_{1} \cdots i_{n}}$.

The next step is to consider intervals $\Delta_{i_{1} \cdots i_{n}}$ with length $r_{i_{1} \cdots i_{n}}$ that may satisfy no asymptotic behavior, and may depend on all the symbolic past.

Received April 5, 1996.

1991 Mathematics Subject Classification 58F15, 28D20, 58F03.

Supported by Program CIÊNCIA, Fellowship BD 2366/92-RM and Program PRAXIS XXI, Fellowship BD 5236/95, JNICT, Portugal, and by a Fulbright/FLAD grant. 
Let $\sigma\left(i_{1} i_{2} \cdots\right)=\left(i_{2} i_{3} \cdots\right)$ be the shift map. If the sequence of functions $\varphi_{n}\left(i_{1} i_{2} \cdots\right)=\log r_{i_{1} \cdots i_{n}}$ is sub-additive, that is, if $\varphi_{n+m} \leq \varphi_{n}+\varphi_{m} \circ \sigma^{n}$ for every $n, m \geq 1$, we can prove, under mild additional assumptions, that the Hausdorff and box dimensions of $F$ satisfy

$$
\operatorname{dim}_{H} F=\underline{\operatorname{dim}}_{B} F=\overline{\operatorname{dim}}_{B} F=s,
$$

where $s$ is the unique root of the equation

$$
\lim _{n \rightarrow \infty} \frac{1}{n} \log \sum_{\left(i_{1} \cdots i_{n}\right)} r_{i_{1} \cdots i_{n}}{ }^{s}=0 .
$$

It is remarkable that this holds for any location of the intervals $\Delta_{i_{1} \cdots i_{n}}$.

We can generalize the above constructions to the case of arbitrary symbolic dynamics. Let $\Sigma_{p}^{+}$be the compact set of one-sided sequences on $p$ symbols, and let $Q \subset \Sigma_{p}^{+}$be a compact shift-invariant subset. We define a set $F=F(Q)$ in a way similar to that in (1), where the union is taken only over the $Q$-admissible tuples $\left(i_{1} \cdots i_{n}\right)$, that is, those tuples such that $\left(i_{1} \cdots i_{n}\right)=\left(j_{1} \cdots j_{n}\right)$ for some $\left(j_{1} j_{2} \cdots\right) \in Q$. In [PeW], Pesin and Weiss showed that if the intervals $\Delta_{i_{1} \cdots i_{n}}$ have length $\prod_{k=1}^{n} \lambda_{i_{k}}$, then the identities in (3) hold when we replace $s$ by the unique root of Bowen's equation

$$
P(s \varphi)=0
$$

Here $\varphi$ is the function defined on $Q$ by $\varphi\left(i_{1} i_{2} \cdots\right)=\log \lambda_{i_{1}}$, and $P$ is the topological pressure with respect to the shift map. We recall that for an arbitrary continuous function $\varphi$ on $Q$, we have

$$
P(s \varphi)=\lim _{n \rightarrow \infty} \frac{1}{n} \log \sum_{\substack{\left(i_{1} \cdots i_{n}\right) \\ Q \text {-admissible }}} \exp \sup \left(s \sum_{k=0}^{n-1} \varphi \circ \sigma^{k}\right),
$$

where the supremum is taken over the sequences $\left(j_{1} j_{2} \cdots\right) \in Q$ such that $\left(j_{1} \cdots j_{n}\right)=\left(i_{1} \cdots i_{n}\right)$. As this example illustrates, we can use the thermodynamic formalism in complicated problems of dimension theory.

In [Bo], Bowen introduced equation (4). It has a rather universal character: most equations used to compute or estimate dimensions are particular cases of it. For example, if $Q=\Sigma_{p}^{+}$then equation (4) is equivalent to equation (2).

We can also consider geometric constructions modeled by arbitrary symbolic dynamics, with intervals $\Delta_{i_{1} \cdots i_{n}}$ with length $r_{i_{1} \cdots i_{n}}$ that may satisfy 
no asymptotic behavior, and may depend on all the symbolic past. If the sequence $\left\{\varphi_{n}\right\}$ defined above is sub-additive, we can prove, under mild additional assumptions, that the identities in (3) hold when we replace $s$ by the unique root of the equation $P\left(s\left\{\varphi_{n}\right\}\right)=0$, where $P$ is an appropriate generalization of the topological pressure. This new pressure may become non-additive, in the sense that the function $\varphi_{n}$ may substitute the sum $\sum_{k=0}^{n-1} \varphi \circ \sigma^{k}$ in (5).

In [Ba1], we develop a non-additive version of the topological pressure, for arbitrary sequences of functions $\left\{\varphi_{n}\right\}$ that may neither satisfy additivity nor even sub-additivity. We follow the approach of Pesin in [P1], to define pressure as a Carathéodory dimension characteristic. The new pressure generalizes the well-known notion of topological pressure on a compact set, introduced by Ruelle in [R1] for expansive maps, and by Walters in [W] in the general case.

Using the non-additive thermodynamic formalism, we unify and extend many results of dimension theory of hyperbolic dynamical systems. In particular, we establish lower and upper dimension estimates for repellers of continuous expanding maps, and for the intersection of basic sets of Axiom $A^{\sharp}$ homeomorphisms with stable and unstable sets. The main elements of our approach are the use of Markov partitions to reproduce the structure of the invariant sets, and a version of Lyapunov exponents for continuous maps, that in the smooth case coincide with the Lyapunov exponents.

In the special case of smooth dynamical systems, we provide a straightforward approach, with proofs entirely based on the thermodynamic formalism. In particular, we recover the formula giving the Hausdorff dimension of basic sets of Axiom A surface diffeomorphisms, established by McCluskey and Manning in $[\mathrm{MM}]$. When we establish the dimension estimates along a stable or unstable set, we need no information about the symbolic dynamics in the transverse direction.

\section{Non-additive thermodynamic formalism}

Non-additive topological pressure. Let $(X, \rho)$ be a compact metric space, and $f: X \rightarrow X$ a continuous map. Let $\mathcal{U}$ be a finite open cover of $X$. We denote by $\mathcal{W}_{n}(\mathcal{U})$ the collection of strings $\mathbf{U}=U_{0} \cdots U_{n}$ of sets $U_{0}, \ldots, U_{n} \in \mathcal{U}$, with length $m(\mathbf{U})=n$. For each $\mathbf{U} \in \mathcal{W}_{n}(\mathcal{U})$, we define the open set $X(\mathbf{U})=\bigcap_{k=0}^{n} f^{-k} U_{k}$. We say that the collection of strings $\Gamma \subset \bigcup_{n \geq 1} \mathcal{W}_{n}(\mathcal{U})$ covers the set $Z \subset X$ if $\bigcup_{\mathbf{U} \in \Gamma} X(\mathbf{U}) \supset Z$.

We consider a sequence of functions $\varphi_{n}: X \rightarrow \mathbb{R}$ and denote it by $\Phi$. For each positive integer $n$, we define

$$
\gamma_{n}(\Phi, \mathcal{U})=\sup \left\{\left|\varphi_{n}(x)-\varphi_{n}(y)\right|: x, y \in X(\mathbf{U}) \text { for some } \mathbf{U} \in \mathcal{W}_{n}(\mathcal{U})\right\} \text {. }
$$


We denote by $|U|=\sup \{\rho(x, y): x, y \in U\}$ the diameter of the set $U \subset X$, and by $|\mathcal{U}|=\sup \{|U|: U \in \mathcal{U}\}$ the diameter of the cover $\mathcal{U}$. We assume that the following property holds:

$$
\varlimsup_{|\mathcal{U}| \rightarrow 0} \varlimsup_{n \rightarrow \infty} \frac{\gamma_{n}(\Phi, \mathcal{U})}{n}=0
$$

We follow the approach in $[\mathrm{P} 1]$ to define pressure as a Carathéodory dimension characteristic. For each string $\mathbf{U} \in \mathcal{W}_{n}(\mathcal{U})$, we write $\varphi(\mathbf{U})=$ $\sup _{X(\mathbf{U})} \varphi_{n}$ when $X(\mathbf{U}) \neq \varnothing$, and $\varphi(\mathbf{U})=-\infty$ otherwise. For each set $Z \subset X$ and $\alpha \in \mathbb{R}$, we define

$$
M(Z, \alpha, \Phi, \mathcal{U})=\lim _{n \rightarrow \infty} \inf _{\Gamma} \sum_{\mathbf{U} \in \Gamma} \exp (-\alpha m(\mathbf{U})+\varphi(\mathbf{U}))
$$

where the infimum is taken over all $\Gamma \subset \bigcup_{k \geq n} \mathcal{W}_{k}(\mathcal{U})$ that covers $Z$. Likewise, we define

$$
\begin{aligned}
& \underline{M}(Z, \alpha, \Phi, \mathcal{U})=\underline{\lim _{n \rightarrow \infty}} \inf _{\Gamma} \sum_{\mathbf{U} \in \Gamma} \exp (-\alpha n+\varphi(\mathbf{U})), \\
& \bar{M}(Z, \alpha, \Phi, \mathcal{U})=\varlimsup_{n \rightarrow \infty} \inf _{\Gamma} \sum_{\mathbf{U} \in \Gamma} \exp (-\alpha n+\varphi(\mathbf{U})),
\end{aligned}
$$

where the infimum is now taken over all $\Gamma \subset \mathcal{W}_{n}(\mathcal{U})$ that covers $Z$. When $\alpha$ goes from $-\infty$ to $+\infty$, each of the quantities in (7), (8), and (9) jumps from $+\infty$ to 0 at a unique critical value. Hence, we can define

$$
\begin{aligned}
P_{Z}(\Phi, \mathcal{U}) & =\inf \{\alpha: M(Z, \alpha, \Phi, \mathcal{U})=0\}, \\
\underline{C P}_{Z}(\Phi, \mathcal{U}) & =\inf \{\alpha: \underline{M}(Z, \alpha, \Phi, \mathcal{U})=0\}, \\
\overline{C P}_{Z}(\Phi, \mathcal{U}) & =\inf \{\alpha: \bar{M}(Z, \alpha, \Phi, \mathcal{U})=0\} .
\end{aligned}
$$

Theorem 1. The following limits exist:

$$
\begin{gathered}
P_{Z}(\Phi) \stackrel{\text { def }}{=} \lim _{|\mathcal{U}| \rightarrow 0} P_{Z}(\Phi, \mathcal{U}), \\
\underline{C P_{Z}}(\Phi) \stackrel{\text { def }}{=} \lim _{|\mathcal{U}| \rightarrow 0} \frac{C P_{Z}}{}(\Phi, \mathcal{U}), \quad \overline{C P}_{Z}(\Phi) \stackrel{\text { def }}{=} \lim _{|\mathcal{U}| \rightarrow 0} \overline{C P}_{Z}(\Phi, \mathcal{U}) .
\end{gathered}
$$

We call $P_{Z}(\Phi)$ the non-additive topological pressure, and $\underline{C P}_{Z}(\Phi)$ and $\overline{C P}_{Z}(\Phi)$ the non-additive lower and upper capacity topological pressures of the sequence of functions $\Phi$ on the set $Z$ (with respect to $f$ ). We emphasize that the set $Z$ need not be compact nor $f$-invariant. 
In the classical thermodynamic formalism, we consider a continuous function $\varphi$ on $X$, and define the sequence of functions $\varphi_{n}=\sum_{k=0}^{n-1} \varphi \circ f^{k}$. Using the uniform continuity of $\varphi$, one can show that property (6) holds. We recover the notion of topological pressure introduced by Pesin and Pitskel' in $[\mathrm{PP}]$, and the notions of lower and upper capacity topological pressures introduced by Pesin in [P1] for arbitrary sets. When $Z=X$, we obtain the topological pressure for compact sets introduced by Ruelle in [R1] in the case of expansive maps, and by Walters in [W] in the general case.

In [Ba1], we establish a non-additive version and generalization of the classical variational principle for the topological pressure.

Bowen's equation. For each real number $s$ we denote by $s \Phi$ the sequence of functions $\left\{s \varphi_{n}\right\}$. Let $h(f)$ be the topological entropy of $f$.

Theorem 2. If $K_{1} n \leq \varphi_{n} \leq K_{2} n$ for all sufficiently large $n$, where $K_{1}, K_{2}$ are negative constants, and $h(f)<\infty$, then there are unique roots $s_{P}, s_{C P}$, and $s_{\overline{C P}}$ of the equations $P_{Z}(s \Phi)=0, \underline{C P}_{Z}(s \Phi)=0$, and $\overline{C P}_{Z}(s \Phi)=0$.

Each equation in Theorem 2 is a non-additive version of Bowen's equation $P(s \varphi)=0$. Here $P$ is the classical topological pressure with respect to some continuous map $f: X \rightarrow X$, and $\varphi$ is a continuous function on $X$. This equation establishes the connection between the thermodynamic formalism and dimension theory: its unique root often gives the exact value or a good estimate for dimension. A good way to think about the relation between dimension and pressure is that both are Carathéodory dimension characteristics (see $[\mathrm{P} 1]$ ).

\section{Applications to dimension theory}

A symbolic geometric construction in $\mathbb{R}^{m}$ is defined by:

(a) a compact shift-invariant set $Q \subset \Sigma_{p}^{+}$for some $p \geq 1$;

(b) a decreasing sequence of compact sets $\Delta_{i_{1} \cdots i_{n}} \subset \mathbb{R}^{m}$ for each sequence $\left(i_{1} i_{2} \cdots\right) \in Q$, with diameter $\left|\Delta_{i_{1} \cdots i_{n}}\right| \rightarrow 0$ as $n \rightarrow \infty$.

The limit set of the construction is the compact set $F=\bigcap_{n=1}^{\infty} \cup \Delta_{i_{1} \cdots i_{n}}$, where the union is taken over all $Q$-admissible $n$-tuples, that is, all tuples $\left(i_{1} \cdots i_{n}\right)$ such that $\left(i_{1} \cdots i_{n}\right)=\left(j_{1} \cdots j_{n}\right)$ for some $\left(j_{1} j_{2} \cdots\right) \in Q$.

We notice that the sets $\Delta_{i_{1} \cdots i_{n}}$ need not be disjoint for any fixed $n$. Our constructions include as trivial examples any iterated function scheme defined by a family of contraction maps. Our setting is much more general and the limit sets that we consider may not be obtained using a family of contraction maps. 
Generalized Moran constructions are constructions such that there exist balls $\underline{B}_{i_{1} \cdots i_{n}}$ and $\bar{B}_{i_{1} \cdots i_{n}}$ for each $\left(i_{1} i_{2} \cdots\right) \in Q$ and $n \geq 1$ such that:

(a) $\underline{B}_{i_{1} \cdots i_{n}} \subset \Delta_{i_{1} \cdots i_{n}} \subset \bar{B}_{i_{1} \cdots i_{n}}$

(b) the interiors of $\underline{B}_{i_{1} \cdots i_{n}}$ and $\underline{B}_{j_{1} \cdots j_{m}}$ are disjoint for any $\left(i_{1} \cdots i_{n}\right) \neq$ $\left(j_{1} \cdots j_{n}\right)$ and $m \geq n$

(c) $\underline{B}_{i_{1} \cdots i_{n}}$ and $\bar{B}_{i_{1} \cdots i_{n}}$ have radii $C_{1} r_{i_{1} \cdots i_{n}}$ and $C_{2} r_{i_{1} \cdots i_{n}}$, for some positive number $r_{i_{1} \cdots i_{n}}$ and positive constants $C_{1} \leq C_{2}$.

We notice that the numbers $r_{i_{1} \cdots i_{n}}$ may depend on all the symbolic past, and may satisfy no asymptotic behavior. A special class of generalized Moran constructions is the class of Moran constructions, introduced in $[\mathrm{M}]$ when $Q=\Sigma_{p}^{+}$(see $\left.[\mathrm{PeW}]\right)$.

We define the sequence of functions $\varphi_{n}\left(i_{1} i_{2} \cdots\right)=\log r_{i_{1} \cdots i_{n}}$ on $Q$, and denote it $\Phi$. Then property (6) holds for the shift map. We assume that there exist $K>1$ and $\delta>0$ such that, for each $\left(i_{1} i_{2} \cdots\right) \in Q$ and $n \geq 1$,

$$
r_{i_{1} \cdots i_{n}} \leq K^{-n} \quad \text { and } \quad r_{i_{1} \cdots i_{n+1}} \geq \delta r_{i_{1} \cdots i_{n}} .
$$

By (10) and Theorem 2, there exist unique roots $s_{P}, s_{C P}$, and $s_{\overline{C P}}$ of the equations $P_{Q}(s \Phi)=0, \underline{C P}_{Q}(s \Phi)=0$, and $\overline{C P}_{Q}(s \Phi)=0$.

Theorem 3. For a generalized Moran construction satisfying (10), we have:

(a) $s_{P} \leq \operatorname{dim}_{H} F \leq \underline{\operatorname{dim}}_{B} F \leq \overline{\operatorname{dim}}_{B} F \leq s_{\overline{C P}}$;

(b) if $\Phi$ is sub-additive then $\operatorname{dim}_{H} F=\underline{\operatorname{dim}}_{B} F=\overline{\operatorname{dim}}_{B} F=s_{P}=$ $s_{\underline{C P}}=s_{\overline{C P}}=s$, where $s$ is the unique root of the equation

$$
\lim _{n \rightarrow \infty} \frac{1}{n} \log \sum_{\substack{\left(i_{1} \cdots i_{n}\right) \\ Q \text {-admissible }}} r_{i_{1} \cdots i_{n}}{ }^{s}=0 .
$$

In [Ba1], we construct an example which demonstrates that we may have strict inequalities in Theorem $3 \mathrm{a}$ if the sequence $\Phi$ is not sub-additive. In [Ba2], we establish extensions of Theorem 3 to classes of limit sets with more complicated geometry.

\section{Dimension of hyperbolic sets}

Repellers of continuous expanding maps. Let $(X, d)$ be a compact metric space. A continuous map $h: X \rightarrow X$ is called expanding if it is a local homeomorphism at every point, and there exist constants $a \geq b>1$ and $r_{0}>0$ such that, for each $x \in X$ and $0<r<r_{0}$, we have

$$
B(h x, b r) \subset h(B(x, r)) \subset B(h x, a r) .
$$


We then say that $X$ is a repeller of $h$. One can show that a continuous expanding map is locally Lipschitz (see (12) below).

Let $R_{1}, \ldots, R_{p}$ be a Markov partition of $X$ with transfer matrix $A$, and $\Sigma_{A}^{+}$the associated topological Markov chain. For each $\omega=\left(i_{1} i_{2} \cdots\right) \in \Sigma_{A}^{+}$ and integers $n, k \geq 1$, we define the lower and upper ratio coefficients respectively by

$$
\begin{aligned}
& \underline{\lambda}_{k}(\omega, n)=\operatorname{mininf}\left\{\frac{d(x, y)}{d\left(h^{n} x, h^{n} y\right)}: x, y \in R_{j_{1} \cdots j_{n+k}}, x \neq y\right\}, \\
& \bar{\lambda}_{k}(\omega, n)=\max \sup \left\{\frac{d(x, y)}{d\left(h^{n} x, h^{n} y\right)}: x, y \in R_{j_{1} \cdots j_{n+k}}, x \neq y\right\},
\end{aligned}
$$

where the minimum and maximum are taken over the $\Sigma_{A}^{+}$-admissible tuples $\left(j_{1} \cdots j_{n+k}\right)$ with $\left(j_{1} \cdots j_{n}\right)=\left(i_{1} \cdots i_{n}\right)$, and $R_{i_{1} \cdots i_{n}}=\bigcap_{k=1}^{n} h^{-k+1} R_{i_{k}}$.

The ratio coefficients play the role of Lyapunov exponents for continuous maps that may not be differentiable. Since the maps $k \mapsto \underline{\lambda}_{k}(\omega, n)$ and $k \mapsto \bar{\lambda}_{k}(\omega, n)$ are respectively non-decreasing and non-increasing for each fixed $\omega \in \Sigma_{A}^{+}$and $n \geq 1$, we can define $\underline{\lambda}(\omega, n)=\lim _{k \rightarrow \infty} \underline{\lambda}_{k}(\omega, n)$ and $\bar{\lambda}(\omega, n)=\lim _{k \rightarrow \infty} \bar{\lambda}_{k}(\omega, n)$. One can show that $\log \underline{\lambda}(\omega, n)$ and $\log \bar{\lambda}(\omega, n)$ are respectively sup-additive and sub-additive cocycles (see [Ba1]). Hence, if $\mu$ is a shift-invariant probability measure on $\Sigma_{A}^{+}$, and $\log ^{-} \underline{\lambda}(\cdot, 1)$ and $\log ^{+} \bar{\lambda}(\cdot, 1)$ are $\mu$-integrable on $\Sigma_{A}^{+}$, then the limits

$$
\underline{\lambda}(\omega)=\lim _{n \rightarrow \infty} \frac{1}{n} \log \underline{\lambda}(\omega, n) \quad \text { and } \quad \bar{\lambda}(\omega)=\lim _{n \rightarrow \infty} \frac{1}{n} \log \bar{\lambda}(\omega, n)
$$

exist for $\mu$-almost every $\omega \in \Sigma_{A}^{+}$. This follows from Kingman's sub-additive ergodic theorem. When $h$ is smooth, the numbers $\underline{\lambda}(\omega)$ and $\bar{\lambda}(\omega)$ respectively coincide with the inverse of the largest and smallest Lyapunov exponents of $h$ at the point $\bigcap_{n=1}^{\infty} h^{-n+1} R_{i_{n}}$, where $\omega=\left(i_{1} i_{2} \cdots\right)$. Related notions were first considered by Kifer in $[\mathrm{K}]$.

We define the sequences of functions

$$
\underline{\varphi}_{n}^{k}(\omega)=\log \underline{\lambda}_{k}(\omega, n) \quad \text { and } \quad \bar{\varphi}_{n}^{k}(\omega)=\log \bar{\lambda}_{k}(\omega, n)
$$

on $\Sigma_{A}^{+}$, and denote them $\underline{\Phi}^{k}$ and $\bar{\Phi}^{k}$. One can show that

$$
a^{-n} \leq \underline{\lambda}_{k}(\omega, n) \leq \bar{\lambda}_{k}(\omega, n) \leq b^{-n}
$$

for each $\omega \in \Sigma_{A}^{+}, n \geq 1$, and all sufficiently large $k \geq 1$.

By (12) and Theorem 2, there exist unique roots $\underline{s}^{k}$ and $\bar{s}^{k}$ of the equations $\overline{C P}_{\Sigma_{A}^{+}}\left(s \underline{\Phi}^{k}\right)=0$ and $P_{\Sigma_{A}^{+}}\left(s \bar{\Phi}^{k}\right)=0$. 
Theorem 4. If $X$ is a repeller of a topologically mixing expanding map then $\underline{s}^{k} \leq \operatorname{dim}_{H} X \leq \underline{\operatorname{dim}}_{B} X \leq \overline{\operatorname{dim}}_{B} X \leq \bar{s}^{k}$.

The map $h$ is called asymptotically conformal if for some $k \geq 1$, given $\varepsilon>0$ we have $\bar{\lambda}_{k}(\omega, n) \leq e^{\varepsilon n} \underline{\lambda}_{k}(\omega, n)$ for all $\omega \in \Sigma_{A}^{+}$and all large $n$.

Theorem 5. If $X$ is a repeller of a topologically mixing expanding map which is asymptotically conformal, then $\operatorname{dim}_{H} X=\underline{\operatorname{dim}}_{B} X=\operatorname{dim}_{B} X=s$, where $s$ is the unique root of the equation

$$
\lim _{n \rightarrow \infty} \frac{1}{n} \log \sum_{\substack{\left(i_{1} \cdots i_{n}\right) \\ \Sigma_{A}^{+} \text {-admissible }}}\left|R_{i_{1} \cdots i_{n}}\right|^{s}=0 .
$$

Repellers of smooth expanding maps. Let $g$ be a $C^{1}$ map on the manifold $M$, and $J$ a compact subset of $M$. We call $g$ expanding and $J$ a repeller of $g$ if:

(a) there exists $a>1$ such that $\left\|d_{x} g u\right\| \geq a\|u\|$ for all $x \in J$ and $u \in T_{x} M$ (for some Riemannian metric on $M$ );

(b) $J=\bigcap_{n>0} g^{-n} V$ for some open neighborhood $V$ of $J$;

(c) $g$ is topologically mixing on $J$.

Then $g J=J$ and $g: J \rightarrow J$ is expanding in the former sense.

A smooth map $g$ is called conformal if $d_{x} g$ is a multiple of an isometry for every point $x \in M$. Examples of repellers of conformal smooth expanding maps are hyperbolic Julia sets and dynamically defined Cantor sets (see $[\mathrm{T}]$ ).

We show in [Ba1] that every conformal $C^{1}$ expanding map is asymptotically conformal. We define the function $\varphi(x)=-\log \left\|d_{x} g\right\|$ on $M$, and let $P_{g}$ be the classical topological pressure with respect to $g$.

Theorem 6. Let $J$ be a repeller of the conformal $C^{1}$ expanding map $g$. Then $\operatorname{dim}_{H} J=\underline{\operatorname{dim}}_{B} J=\overline{\operatorname{dim}}_{B} J=s$, where $s$ is the unique root of Bowen's equation $P_{g}(s \varphi)=0$.

In [R2], Ruelle proved that the Hausdorff dimension of a repeller of a conformal $C^{1+\alpha}$ expanding map is given by Bowen's equation. In $[\mathrm{F}]$, Falconer showed that the Hausdorff and box dimensions of these repellers coincide. The statement of Theorem 6 was also established by Gatzouras and Peres in $[\mathrm{GP}]$, and by Takens in $[\mathrm{T}]$ for dynamically defined Cantor sets.

Basic sets of Axiom $\mathbf{A}^{\sharp}$ homeomorphisms. Let $f$ be a homeomorphism of the compact metric space $(X, d)$. Given $\varepsilon>0$, we define the local 
stable and unstable sets at the point $x \in X$ respectively by

$$
\begin{aligned}
& W^{s}(x)=\left\{y \in X: d\left(f^{n} y, f^{n} x\right) \leq \varepsilon \text { for all } n \geq 0\right\}, \\
& W^{u}(x)=\left\{y \in X: d\left(f^{n} y, f^{n} x\right) \leq \varepsilon \text { for all } n \leq 0\right\} .
\end{aligned}
$$

We say that $f$ is an Axiom $A^{\sharp}$ homeomorphism (see [AY]) if there exist constants $\lambda \in(0,1)$ and $\varepsilon, \delta>0$ such that:

(a) for each $x \in X$ and $n \geq 0$, we have $d\left(f^{n} y, f^{n} z\right) \leq \lambda^{n} d(y, z)$ for all $y, z \in W^{s}(x)$, and $d\left(f^{-n} y, f^{-n} z\right) \leq \lambda^{n} d(y, z)$ for all $y, z \in W^{u}(x)$;

(b) for every $x, y \in X$ with $d(x, y)<\delta$, the set $W^{s}(x) \cap W^{u}(y)$ consists of a single point, which we denote $[x, y]$. We require the map $(x, y) \mapsto[x, y]$ to be continuous.

Then the set of non-wandering points is a finite union of disjoint closed $f$-invariant sets, called basic sets.

Let $f$ be an Axiom $\mathrm{A}^{\sharp}$ homeomorphism and $\Lambda \subset X$ a basic set. We consider a Markov partition $R_{1}, \ldots, R_{p}$ of $\Lambda$ with transfer matrix $A$, and write $R_{i_{0} \cdots i_{n}}=\bigcap_{k=0}^{n} f^{-k} R_{i_{k}}$. Fix $x \in \Lambda$. For each $\omega=\left(i_{0} i_{1} \cdots\right) \in \Sigma_{A}^{+}$ and integers $n, k \geq 1$, we define

$$
\begin{aligned}
& \underline{\lambda}_{k}^{u}(\omega, n)=\operatorname{mininf}\left\{\frac{d(y, z)}{d\left(f^{n} y, f^{n} z\right)}: y, z \in W^{u}(x) \cap R_{j_{0} \cdots j_{n+k}}, y \neq z\right\}, \\
& \bar{\lambda}_{k}^{u}(\omega, n)=\max \sup \left\{\frac{d(y, z)}{d\left(f^{n} y, f^{n} z\right)}: y, z \in W^{u}(x) \cap R_{j_{0} \cdots j_{n+k}}, y \neq z\right\},
\end{aligned}
$$

where the minimum and maximum are taken over the $\Sigma_{A}^{+}$-admissible tuples $\left(j_{0} \cdots j_{n+k}\right)$ such that $\left(j_{0} \cdots j_{n}\right)=\left(i_{0} \cdots i_{n}\right)$. We assume that

$$
\text { the map } f \mid W^{u}(x) \text { is expanding for every } x \in \Lambda \text {, }
$$

that is, the map $f \mid W^{u}(x): W^{u}(x) \rightarrow f W^{u}(x)$ satisfies the inclusions in (11), with $h$ substituted by $f$, for some constants $a(x) \geq b(x)>1$. Furthermore, we assume that $\overline{\lim }_{n \rightarrow \infty}\left(a\left(f^{n} x\right) \cdots a(x)\right)^{1 / n}$ is finite for every $x \in \Lambda$.

Fix $x \in \Lambda$. We define the sequences of functions $\underline{\varphi}_{n}^{k}(\omega)=\log \underline{\lambda}_{k}^{u}(\omega, n)$ and $\bar{\varphi}_{n}^{k}(\omega)=\log \bar{\lambda}_{k}^{u}(\omega, n)$ on $\Sigma_{A}^{+}$. By Theorem 2 and (13), there exist unique roots $\underline{s}^{k}(x)$ and $\bar{s}^{k}(x)$ of the equations $\overline{C P}_{\Sigma_{A}^{+}}\left(s \underline{\Phi}^{k}\right)=0$ and $P_{\Sigma_{A}^{+}}\left(s \bar{\Phi}^{k}\right)=0$.

Theorem 7. Let $\Lambda$ be a basic set of a topologically mixing Axiom $A^{\sharp}$ homeomorphism. Then $\underline{s}^{k}(x) \leq \operatorname{dim}_{H} W^{u}(x) \cap \Lambda \leq \underline{\operatorname{dim}}_{B} W^{u}(x) \cap \Lambda \leq$ $\overline{\operatorname{dim}}_{B} W^{u}(x) \cap \Lambda \leq \bar{s}^{k}(x)$. 
Property (13) is automatically satisfied when $f$ is of class $C^{1}$, but it may not hold for arbitrary Axiom $A^{\sharp}$ homeomorphisms. More generally, if the map $f$ is locally Lipschitz, then property (13) holds.

In a similar way, we can obtain dimension estimates for the stable sets. Basic sets of Axiom A diffeomorphisms. Let $f$ be a $C^{1}$ diffeomorphism on the manifold $M$. An $f$-invariant set $\Lambda \subset M$ is called hyperbolic if the tangent bundle $T_{\Lambda} M$ splits into stable and unstable bundles $E^{s}$ and $E^{u}$, invariant under $d f$. We say that $f$ is an Axiom A diffeomorphism if the set of non-wandering points is hyperbolic and is the closure of the set of periodic points of $f$. We define the functions $\varphi^{s}(x)=\log \left\|d_{x} f \mid E^{s}\right\|$ and $\varphi^{u}(x)=-\log \left\|d_{x} f \mid E^{u}\right\|$ on $\Lambda$. Let $t^{s}$ and $t^{u}$ be the unique roots of the equations $P_{f}\left(t \varphi^{s}\right)=0$ and $P_{f}\left(t \varphi^{u}\right)=0$.

Theorem 8. Let $\Lambda$ be a basic set of a $C^{1}$ Axiom A surface diffeomorphism, with $\operatorname{dim} E^{s}=\operatorname{dim} E^{u}=1$. Then $\operatorname{dim}_{H} \Lambda=\underline{\operatorname{dim}}_{B} \Lambda=\overline{\operatorname{dim}}_{B} \Lambda=t^{s}+t^{u}$.

In $[\mathrm{MM}]$, McCluskey and Manning proved that $\operatorname{dim}_{H} \Lambda=t^{s}+t^{u}$. The coincidence of the Hausdorff and box dimensions was previously shown by Takens in the case of $C^{2}$ diffeomorphisms (see [T]), and by Palis and Viana in $[\mathrm{PV}]$ for arbitrary $C^{1}$ diffeomorphisms. The technique in $[\mathrm{PV}]$ differs from the one in [Ba1] and is not based on the thermodynamic formalism. In higher dimensional manifolds, the Hausdorff and box dimensions of basic sets may not coincide (see $[\mathrm{PoW}]$ for examples).

In [Ba1], we also obtain lower and upper dimension estimates for basic sets in higher dimensional manifolds. In [P2], Pesin gives a straightforward treatment of the case where one of the maps $d f \mid E^{u}$ and $d f \mid E^{s}$ is conformal.

\section{References}

[AY] V. Alekseev and M. Yakobson, Symbolic dynamics and hyperbolic dynamic systems, Phys. Rep. 75 (1981), no. 5, 287-325.

[Ba1] L. Barreira, A non-additive thermodynamic formalism and applications to dimension theory of hyperbolic dynamical systems, Ergodic Theory Dynam. Systems (to appear).

[Ba2] - Dimension of Cantor sets with complicated geometry, Equadiff 95 Proceedings (to appear).

[Bo] R. Bowen, Hausdorff dimension of quasi-circles, Inst. Hautes Études Sci. Publ. Math. 50 (1979), 259-273.

[F] K. Falconer, Dimensions and measures of quasi self-similar sets, Proc. Amer. Math. Soc. 106 (1989), 543-554.

[GP] D. Gatzouras and Y. Peres, Invariant measures of full dimension for some expanding maps, Preprint (1994).

[K] Y. Kifer, Characteristic exponents of dynamical systems in metric spaces, Ergodic Theory Dynam. Systems 3 (1983), 119-127.

[MM] H. McCluskey and A. Manning, Hausdorff dimension for horseshoes, Ergodic Theory Dynam. Systems 3 (1983), 251-260. 
[M] P. Moran, Additive functions of intervals and Hausdorff measure, Math. Proc. Cambridge Philos. Soc. 42 (1946), 15-23.

[PV] J. Palis and M. Viana, On the continuity of Hausdorff dimension and limit capacity for horseshoes, Dynamical Systems, Valparaiso 1986, Springer Lecture Notes in Mathematics (R. Bamón, R. Labarca and J. Palis Jr., eds.), vol. 1331, Springer Verlag, 1988, pp. 150-160.

[P1] Ya. Pesin, Dimension type characteristics for invariant sets of dynamical systems, Russian Math. Surveys 43 (1988), 111-151.

[P2] - Dimension theory in dynamical systems: contemporary view and applications, Chicago University Press (to appear).

[PP] Ya. Pesin and B. Pitskel', Topological pressure and the variational principle for noncompact sets, Functional Anal. Appl. 18 (1984), 307-318.

$[\mathrm{PeW}]$ Ya. Pesin and $\mathrm{H}$. Weiss, On the dimension of deterministic and random Cantorlike sets, symbolic dynamics, and the Eckmann-Ruelle conjecture, Comm. Math. Phys. (to appear).

[PoW] M. Pollicott and H. Weiss, The dimensions of some self affine limit sets in the plane and hyperbolic sets, J. Statist. Phys. 77 (1994), 841-866.

[R1] D. Ruelle, Statistical mechanics on a compact set with $\mathbb{Z}^{\nu}$ action satisfying expansiveness and specification, Trans. Amer. Math. Soc. 185 (1973), 237-251.

[R2] Repellers for real analytic maps, Ergodic Theory Dynam. Systems 2 (1982), 99-107.

[T] F. Takens, Limit capacity and Hausdorff dimension of dynamically defined Cantor sets, Dynamical Systems, Valparaiso 1986, Springer Lecture Notes in Mathematics (R. Bamón, R. Labarca and J. Palis Jr., eds.), vol. 1331, Springer Verlag, 1988, pp. 196-212.

[W] P. Walters, A variational principle for the pressure of continuous transformations, Amer. J. Math. 97 (1976), 937-971.

Departamento de M atemática, Instituto Superior Téchico, 1096 Lisboa CODEX, PORTUGAL

Current address: Department of Mathematics, Pennsylvania State University, University Park, PA 16802, U.S.A.

E-mail address: luis@math.psu.edu, barreira@math.ist.utl.pt 